\title{
The ANTARES Optical Beacon System
}

\author{
M. Ageron ${ }^{\mathrm{e}}$, J.A. Aguilar ${ }^{\mathrm{j}, *}$, A. Albert ${ }^{\mathrm{u}}$, F. Ameli ${ }^{\mathrm{x}}$, \\ M. Anghinolfi ${ }^{\mathrm{i}}$, G. Anton ${ }^{\mathrm{g}}, \mathrm{S}$. Anvar ${ }^{\mathrm{y}}, \mathrm{F}$. Ardellier-Desages ${ }^{\mathrm{y}}$, \\ E. Aslanides ${ }^{\mathrm{e}}$, J-J. Aubert ${ }^{\mathrm{e}}$, R. Auer ${ }^{\mathrm{g}}$, E. Barbarito ${ }^{\mathrm{b}}$, \\ S. Basa ${ }^{\mathrm{r}}$, M. Battaglieri ${ }^{\mathrm{i}}$, Y. Becherini ${ }^{\mathrm{c}, 1}$, J. Beltramelli ${ }^{\mathrm{y}}$, \\ V. Bertin ${ }^{\mathrm{e}}$, A. Bigi ${ }^{\mathrm{w}}$, M. Billault ${ }^{\mathrm{e}}, \mathrm{R}$. Blaes ${ }^{\mathrm{u}}, \mathrm{N}$. de Botton ${ }^{\mathrm{y}}$, \\ M.C. Bouwhuis ${ }^{\mathrm{v}}$, S.M. Bradbury ${ }^{\mathrm{t}}$, R. Bruijn ${ }^{\mathrm{v}, \mathrm{ab}}$, J. Brunner ${ }^{\mathrm{e}}$, \\ G.F. Burgio ${ }^{f}$, J. Busto ${ }^{\text {e }}$, F. Cafagna ${ }^{b}$, L. Caillat ${ }^{\text {e }}$, A. Calzas ${ }^{\text {e }}$, \\ A. Capone ${ }^{\mathrm{x}}$, L. Caponetto ${ }^{\mathrm{f}}$, E. Carmona ${ }^{\mathrm{j}}$, J. Carr ${ }^{\mathrm{e}}$, \\ S.L. Cartwright ${ }^{\mathrm{z}}$, D. Castel ${ }^{\mathrm{u}}$, E. Castorina ${ }^{\mathrm{w}}$, V. Cavasinni ${ }^{\mathrm{w}}$, \\ S. Cecchini ${ }^{\mathrm{c}, \mathrm{m}}, \mathrm{A}$. Ceres ${ }^{\mathrm{b}}, \mathrm{P}$. Charvis ${ }^{\mathrm{h}}, \mathrm{P}$. Chauchot ${ }^{\mathrm{k}}$, \\ T. Chiarusi ${ }^{\mathrm{x}}$, M. Circella $^{\mathrm{b}}$, C. Colnard ${ }^{\mathrm{v}}$, C. Compère ${ }^{\mathrm{k}}$, \\ R. Coniglione ${ }^{\mathrm{s}}$, N. Cottini ${ }^{\mathrm{w}, 1}, \mathrm{P}$. Coyle $^{\mathrm{e}}, \mathrm{S}$. Cuneo ${ }^{\mathrm{i}}$, \\ A-S. Cussatlegras ${ }^{\mathrm{d}}$, G. Damy ${ }^{\mathrm{k}}$, R. van Dantzig ${ }^{\mathrm{v}}$, G. De Bonis ${ }^{\mathrm{x}}$, \\ C. De Marzo ${ }^{\text {b,2 }}$, R. De Vita ${ }^{\mathrm{i}}$, I. Dekeyser ${ }^{\mathrm{d}}$, E. Delagnes ${ }^{\mathrm{y}}$, \\ D. Denans ${ }^{\mathrm{y}}$, A. Deschamps ${ }^{\text {h}}$, J-J. Destelle $^{\mathrm{e}}$, B. Dinkespieler ${ }^{\mathrm{e}}$, \\ C. Distefano ${ }^{\mathrm{s}}$, C. Donzaud ${ }^{\mathrm{y}, 3}, \mathrm{~J}-\mathrm{F}$. Drogou ${ }^{\ell}, \mathrm{F}$. Druillole ${ }^{\mathrm{y}}$, \\ D. Durand ${ }^{\mathrm{y}}$, J-P. Ernenwein ${ }^{\mathrm{u}}$, S. Escoffier ${ }^{\mathrm{e}}$, E. Falchini ${ }^{\mathrm{w}}$, \\ S. Favard ${ }^{\mathrm{e}}$, F. Fehr ${ }^{\mathrm{g}}$, F. Feinstein ${ }^{\mathrm{e}}$, S. Ferry ${ }^{\mathrm{n}}$, C. Fiorello $^{\mathrm{b}}$, \\ V. Flaminio $^{\mathrm{w}}$, K. Fratini ${ }^{\mathrm{i}}$, J-L. Fuda ${ }^{\mathrm{d}}$, S. Galeotti ${ }^{\mathrm{w}}$, \\ J-M. Gallone ${ }^{\mathrm{n}}$, G. Giacomelli ${ }^{\mathrm{c}}$, N. Girard ${ }^{\mathrm{u}}$, C. Gojak ${ }^{\mathrm{e}}$, \\ Ph. Goret ${ }^{y}$, K. Graf ${ }^{g}$, G. Hallewell ${ }^{\mathrm{e}}$, M.N. Harakeh ${ }^{\mathrm{q}}$, \\ B. Hartmann ${ }^{\mathrm{g}}$, A. Heijboer ${ }^{\mathrm{v}, \mathrm{ab}}$, E. Heine ${ }^{\mathrm{v}}$, Y. Hello ${ }^{\mathrm{h}}$, \\ J.J. Hernández-Rey ${ }^{j}$, J. Hößl ${ }^{g}$, C. Hoffman ${ }^{n}$, J. Hogenbirk ${ }^{\mathrm{v}}$, \\ J.R. Hubbard ${ }^{\mathrm{y}}$, M. Jaquet ${ }^{\mathrm{e}}$, M. Jaspers ${ }^{\mathrm{v}, \mathrm{ab}}$, M. de Jong ${ }^{\mathrm{v}}$, \\ F. Jouvenot ${ }^{\mathrm{y}, 4}$, N. Kalantar-Nayestanaki ${ }^{\mathrm{q}}$, A. Kappes ${ }^{\mathrm{g}}$, \\ T. Karg ${ }^{\mathrm{g}}$, U. Katz ${ }^{\mathrm{g}}$, P. Keller ${ }^{\mathrm{e}}$, E. Kok ${ }^{\mathrm{v}}$, H. Kok ${ }^{\mathrm{v}}$, \\ P. Kooijman ${ }^{\text {v,aa }}$, C. Kopper ${ }^{\mathrm{g}}$, E.V. Korolkova ${ }^{\mathrm{z}}$, A. Kouchner ${ }^{\mathrm{a}}$, \\ W. Kretschmer ${ }^{\mathrm{g}}$, A. Kruijer ${ }^{\mathrm{v}}$, S. Kuch ${ }^{\mathrm{g}}$, V.A. Kudryavstev ${ }^{\mathrm{z}}$, \\ P. Lagier ${ }^{\mathrm{e}}$, R. Lahmann ${ }^{\mathrm{g}}$, G. Lamanna ${ }^{\mathrm{e}}$, L. Lamare $^{\mathrm{y}}$, \\ G. Lambard ${ }^{\mathrm{e}}$, J-C. Languillat ${ }^{\mathrm{y}}, \mathrm{H}$. Laschinsky ${ }^{\mathrm{g}}$, J. Lavalle ${ }^{\mathrm{e}}$, \\ Y. Le Guen ${ }^{k}$, H. Le Provost ${ }^{\mathrm{y}}$, A. Le Van Suu ${ }^{\mathrm{e}}$, D. Lefèvre ${ }^{\mathrm{d}}$, \\ T. Legou ${ }^{\mathrm{e}}$, G. Lelaizant ${ }^{\mathrm{e}}, \mathrm{G} \cdot \operatorname{Lim}^{\mathrm{v}, \mathrm{ab}}$, D. Lo Presti ${ }^{\mathrm{f}}$,
}


H. Loehner ${ }^{\mathrm{q}}$, S. Loucatos ${ }^{\mathrm{y}}$, F. Louis ${ }^{\mathrm{y}}$, F. Lucarelli ${ }^{\mathrm{x}}$, V. Lyashuk ${ }^{\mathrm{p}}$, M. Marcelin ${ }^{\mathrm{r}}$, A. Margiotta $^{\mathrm{c}}$, R. Masullo ${ }^{\mathrm{x}}$, F. Mazéas ${ }^{\mathrm{k}}$, A. Mazure $^{\mathrm{r}}$, J.E. McMillan ${ }^{\mathrm{z}}$, R. Megna ${ }^{\mathrm{b}}$, M. Melissas ${ }^{\mathrm{e}}$, E. Migneco $^{\mathrm{s}}$, A. Milovanovic ${ }^{\mathrm{t}}$, M. Mongelli ${ }^{\mathrm{b}}$, T. Montaruli ${ }^{\mathrm{b}, 5}$, M. Morganti $^{\mathrm{w}}$, L. Moscoso $^{\mathrm{y}, \mathrm{a}}$, M. Musumeci $^{\mathrm{s}}$, M. Naumann-Godo ${ }^{g}$, C. Naumann ${ }^{g}$, V. Niess ${ }^{\mathrm{e}}$, T. Noble $^{\mathrm{e}}$, C. Olivetto ${ }^{n}$, R. Ostasch ${ }^{\mathrm{g}}$, N. Palanque-Delabrouille ${ }^{\mathrm{y}}$, P. Payre ${ }^{\mathrm{e}}$, H. Peek ${ }^{\mathrm{v}}$, A. Perez ${ }^{\mathrm{j}}$, C. Petta $^{\mathrm{f}}{ }$, P. Piattelli ${ }^{\mathrm{s}}$, R. Pillet ${ }^{\mathrm{h}}$, J-P. Pineau ${ }^{\mathrm{n}}$, J. Poinsignon ${ }^{\mathrm{y}}, \mathrm{V}$. Popa ${ }^{ }$, T. Pradier ${ }^{\mathrm{n}}$, C. Racca $^{\mathrm{n}}$, N. Randazzo ${ }^{\mathrm{f}}$, J. van Randwijk ${ }^{\mathrm{v}}$,

D. Real ${ }^{\mathrm{j}}$, B. van Rens ${ }^{\mathrm{v}}, \mathrm{F}$. Réthorée ${ }^{\mathrm{e}}, \mathrm{P}$. Rewiersma ${ }^{\mathrm{v}, 2}$,

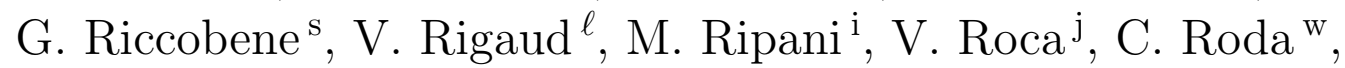
J.F. Rolin ${ }^{\mathrm{k}}$, H.J. Rose ${ }^{\mathrm{t}}$, A. Rostovtsev ${ }^{\mathrm{p}}$, J. Roux ${ }^{\mathrm{e}}$, M. Ruppi $^{\mathrm{b}}$, G.V. Russo ${ }^{f}$, G. Rusydi ${ }^{q}$, F. Salesa ${ }^{j}$, K. Salomon ${ }^{\mathrm{g}}$, P. Sapienza ${ }^{\text {s }}$, F. Schmitt ${ }^{g}$, J-P. Schuller ${ }^{y}$, R. Shanidze ${ }^{g}$, I. Sokalski ${ }^{b}$, T. Spona ${ }^{\mathrm{g}}$, M. Spurio ${ }^{\mathrm{c}}$, G. van der Steenhoven ${ }^{\mathrm{v}}$, T. Stolarczyk ${ }^{y}$, K. Streeb ${ }^{g}$, L. Sulak ${ }^{\mathrm{e}}$, M. Taiuti ${ }^{\mathrm{i}}$, C. Tamburini ${ }^{\mathrm{d}}$, C. Tao $^{\mathrm{e}}$, G. Terreni ${ }^{\mathrm{w}}$, L.F. Thompson ${ }^{\mathrm{z}}$,

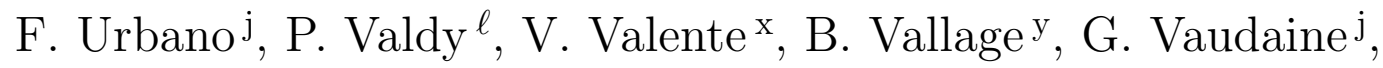
G. Venekamp ${ }^{\mathrm{v}}$, B. Verlaat ${ }^{\mathrm{v}}, \mathrm{P}$. Vernin ${ }^{\mathrm{y}}$, G. de Vries-Uiterweerd ${ }^{\mathrm{v}, \mathrm{aa}}$, R. van Wijk ${ }^{\mathrm{v}}$, G. Wijnker ${ }^{\mathrm{v}}$, P. de Witt Huberts ${ }^{\mathrm{v}}$, G. Wobbe ${ }^{\mathrm{g}}$, E. de Wolf ${ }^{\mathrm{v}, \mathrm{ab}}$, A-F. Yao ${ }^{\mathrm{d}}$, D. Zaborov ${ }^{p}$, H. Zaccone $^{\mathrm{y}}$, J.D. Zornoza ${ }^{\mathrm{j}}$, J. Zúñiga $^{\mathrm{j}}$ 
a APC - AstroParticule et Cosmologie, 10, rue Alice Domon et Léonie Duquet 75205 Paris Cedex 13, France

${ }^{\mathrm{b}}$ Dipartimento Interateneo di Fisica e Sezione INFN, Via E. Orabona 4, 70126 Bari, Italy

${ }^{\mathrm{c}}$ Dipartimento di Fisica dell'Università e Sezione INFN, Viale Berti Pichat 6/2, 40127 Bologna, Italy

${ }^{\mathrm{d}}$ COM - Centre d'Océanologie de Marseille, CNRS/INSU et Université de la Méditerranée, 163 Avenue de Luminy, Case 901, 13288 Marseille Cedex 9, France

${ }^{\mathrm{e}}$ CPPM - Centre de Physique des Particules de Marseille, CNRS/IN2P3 et

Université de la Méditerranée, 163 Avenue de Luminy, Case 902, 13288 Marseille Cedex 9, France

${ }^{\mathrm{f}}$ Dipartimento di Fisica ed Astronomia dell'Università e Sezione INFN, Viale Andrea Doria 6, 95125 Catania, Italy

${ }^{\mathrm{g}}$ Friedrich-Alexander-Universität Erlangen-Nürnberg, Physikalisches Institut, Erwin-Rommel-Str. 1, D-91058 Erlangen, Germany

${ }^{\mathrm{h}}$ GéoSciences Azur, CNRS/INSU, IRD, Université de Nice Sophia-Antipolis, Université Pierre et Marie Curie - Observatoire Océanologique de Villefranche, BP48, 2 quai de la Darse, 06235 Villefranche-sur-Mer Cedex, France

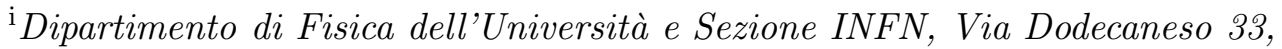
16146 Genova, Italy

jIFIC - Instituto de Física Corpuscular, Edificios de Investigación de Paterna, CSIC - Universitat de València, Apdo. de Correos 22085, 46071 Valencia, Spain

${ }^{\mathrm{k}}$ IFREMER - Centre de Brest, BP 70, 29280 Plouzané, France

${ }^{\ell}$ IFREMER - Centre de Toulon/La Seyne Sur Mer, Port Brégaillon, Chemin Jean-Marie Fritz, 83500, La Seyne sur Mer, France

${ }^{\mathrm{m}}$ INAF-IASF, via P. Gobetti 101, 40129 Bologna, Italy

${ }^{\mathrm{n}}$ IPHC - Institut Pluridisciplinaire Hubert Curien, Université Louis Pasteur (Strasbourg 1) et IN2P3/CNRS, 23 rue du Loess, BP 28, 67037 Strasbourg Cedex 2, France

o Institute for Space Sciences, R-77125 Bucharest-Măgurele, Romania. ${ }^{\mathrm{p}}$ ITEP - Institute for Theoretical and Experimental Physics,

B. Cheremushkinskaya 25, 117259 Moscow, Russia

${ }^{\mathrm{q}}$ Kernfysisch Versneller Instituut (KVI), University of Groningen, Zernikelaan 25, 9747 AA Groningen, The Netherlands

${ }^{\mathrm{r}} L A M$ - Laboratoire d'Astrophysique de Marseille, CNRS/INSU et Université de Provence, Traverse du Siphon - Les Trois Lucs, BP 8, 13012 Marseille Cedex 12, France

${ }^{\mathrm{s} I N F N ~-~ L a b a r a t o r i ~ N a z i o n a l i ~ d e l ~ S u d ~(L N S), ~ V i a ~ S . ~ S o f i a ~ 44, ~} 95123$ Catania, Italy

${ }^{\mathrm{t}}$ School of Physics \& Astronomy, University of Leeds LS2 9JT, UK

${ }^{\mathrm{u}}$ GRPHE - Groupe de Recherche en Physique des Hautes Energies, Université de

Haute Alsace, 61 Rue Albert Camus, 68093 Mulhouse Cedex, France

${ }^{\vee}$ Nationaal Instituut voor Kernfysica en Hoge-Energiefysica (NIKHEF), Kruislaan 409, 1098 SJ Amsterdam, The Netherlands

${ }^{\mathrm{w}}$ Dipartimento di Fisica dell'Università e Sezione INFN, Largo B. Pontecorvo 3, 56127 Pisa, Italy 


\begin{abstract}
“Dipartimento di Fisica dell'Università "La Sapienza" e Sezione INFN, P.le Aldo Moro 2, 00185 Roma, Italy

y DSM/Dapnia - Direction des Sciences de la Matière, laboratoire de recherche sur les lois fondamentales de l'Univers, CEA Saclay, 91191 Gif-sur-Yvette Cedex, France

${ }^{z}$ Dept. of Physics and Astronomy, University of Sheffield, Sheffield S3 7RH, UK

${ }^{\text {aa } U n i v e r s i t e i t ~ U t r e c h t, ~ F a c u l t e i t ~ B e t a w e t e n s c h a p p e n, ~ P r i n c e t o n p l e i n ~ 5, ~} 3584$ CC

Utrecht, The Netherlands

${ }^{\mathrm{ab}}$ Universiteit van Amsterdam, Instituut voor Hoge-Energiefysica, Kruislaan 409, 1098 SJ Amsterdam, The Netherlands
\end{abstract}

\begin{abstract}
ANTARES is a neutrino telescope being deployed in the Mediterranean Sea. It consists of a three dimensional array of photomultiplier tubes that can detect the Cherenkov light induced by charged particles produced in the interactions of neutrinos with the surrounding medium. High angular resolution can be achieved, in particular when a muon is produced, provided that the Cherenkov photons are detected with sufficient timing precision. Considerations of the intrinsic time uncertainties stemming from the transit time spread in the photomultiplier tubes and the mechanism of transmission of light in sea water lead to the conclusion that a relative time accuracy of the order of $0.5 \mathrm{~ns}$ is desirable. Accordingly, different time calibration systems have been developed for the ANTARES telescope. In this article, a system based on Optical Beacons, a set of external and well-controlled pulsed light sources located throughout the detector, is described. This calibration system takes into account the optical properties of sea water, which is used as the detection volume of the ANTARES telescope. The design, tests, construction and first results of the two types of beacons, LED and laser-based, are presented.
\end{abstract}

Key words: neutrino telescope, time calibration, optical beacon PACS: 95.55.Vj, 95.85.Ry

\footnotetext{
* Corresponding author Email address: J.A.Aguilar@ific.uv.es (J.A. Aguilar).

1 Now at: $\mathrm{y}$

2 Deceased.

3 Also at: Orsay - Université Paris-Sud, CNRS-IN2P3, Institut de Physique Nucléaire (UMR 8608) ORSAY, F-91406, France

4 Now at: University of Liverpool, Dept. of Physics, UK

5 On leave at University of Wisconsin - Madison, 53706, WI, USA
} 


\section{Introduction}

The ANTARES telescope is an underwater neutrino detector being deployed in the Mediterranean Sea at a depth of $2500 \mathrm{~m}$ offshore from Toulon (France) [1]. This detector, which is a first step toward a $\mathrm{km}^{3}$-scale undersea neutrino telescope, will consist of twelve lines with a sensitive area for high energy muons of more than $0.05 \mathrm{~km}^{2}$ for $\mathrm{E}_{\mu}>100 \mathrm{TeV}$. The construction of the ANTARES neutrino telescope started with the installation of the first lines in 2006, and the detector is scheduled to be completed by the end of 2007. In addition, a special instrumentation line, the MILOM [2], is in operation since Spring 2005. The detection principle and the main detector components are briefly described in section 2. The precision required in the time determination and the different time calibration systems are reviewed in section 3 . The concept of an Optical Beacon system is briefly introduced and the actual solution adopted by ANTARES is reviewed in section 4. Detailed descriptions of the LED and Laser Beacon calibration systems are given in section 5 and 6, respectively. Some results from the first data taken with the lines in operation in 2006 are given in section 7 . Finally, section 8 presents the summary and conclusions.

\section{The ANTARES Neutrino Telescope}

The ANTARES neutrino telescope uses sea water as the detection medium to look for extra-terrestrial neutrinos. Most of these neutrinos cross right through the Earth without interacting. A small fraction of the incoming neutrino flux, however, interacts with the nucleons that make up the matter surrounding the detector. In a charged current interaction a high energy muon neutrino produces a muon which induces Cherenkov light when crossing a suitable optical medium such as ice or water. Other signatures can also be detected.

In order to detect and reconstruct the wavefront of the Cherenkov light, ANTARES is equipped with 900 Optical Modules (OMs). The OM, the basic optical unit of ANTARES, consists of a photomultiplier tube (PMT) housed in a water-pressure resistant glass sphere [3]. An exhaustive study of PMTs was carried out during the R\&D phase which led to the selection of the 14stage, 10" Hamamatsu R7081-20 model [4]. Together with the PMT there is an internal LED for calibration purposes inside the OM. Each group of three OMs constitutes a storey. All the electronics for one storey are housed in a pressure resistant titanium container making up the so-called Local Control Module (LCM). Every OM is read out by an electronics board housed in the LCM carrying a pair of Analogue Ring Samplers (ARS), the ASIC chip used for signal processing and digitisation [5]. The ARS provides the time and amplitude of the signal, both of which are essential to reconstruct the muon track 
direction and estimate its energy.

\section{The ANTARES time calibration systems}

ANTARES is expected to achieve very good angular resolution $\left(<0.3^{\circ}\right.$ for muon events above $10 \mathrm{TeV}$ ). This pointing accuracy is closely related to the precision in the determination of the arrival time of the Cherenkov photons at the PMTs. The relative time resolution between OMs is, therefore, of utmost importance. It is limited by the transit time spread (TTS) of the signal in the PMTs $(\sigma \sim 1.3 \mathrm{~ns})$ and by the scattering and chromatic dispersion of light in sea water $(\sigma \sim 1.5 \mathrm{~ns}$ for a light propagation of $40 \mathrm{~m})$ [6]. The electronics of the ANTARES detector is designed in order to contribute less than $0.5 \mathrm{~ns}$ to the overall time resolution. Therefore, the time calibration should aim at a precision below the nanosecond level. To this end, several complementary time calibration systems are implemented in the ANTARES detector in order to measure and monitor the relative times between different components of the detector due to, e.g. cable lengths and electronics delays. These time calibrations are performed by the following systems:

(1) The internal clock calibration system. A very precise time reference clock distribution system has been implemented in the ANTARES detector. It consists of a $20 \mathrm{MHz}$ clock generator on shore, a clock distribution system and a clock signal transceiver board placed in each LCM. A common clock signal is provided to the ARSs. Synchronised data commands can be superimposed on the clock signal, in particular start and stop commands, which together with a high precision Time to Digital Converter (TDC) make up the essential components of the system. This system also includes an echo-based time calibration whereby each LCM clock electronics board is able to send back a return signal through the same optical path as the outgoing clock signals. This system enables the time offsets between all LCM clock boards to be measured by recording the propagation delays of the return signals of each storey with respect to the original clock signal emission time. Measurements in real conditions show a resolution of $\sim 0.1 \mathrm{~ns}$, well within the specifications. The system also includes the synchronisation with respect to Universal Time, by assigning the GPS timestamp to the data, with a precision of about $100 \mu \mathrm{s}$, much better than the required precision of $\sim 1 \mathrm{~ms}$. The clock signals are distributed across all detector components from the shore up to the clock boards. The remaining path between these boards and the PMT photocathodes however requires a different timing calibration mechanism.

(2) The internal Optical Module LEDs. Inside each Optical Module there is a blue LED attached to the back of the PMT capable of illuminating the photocathode. The LED is an HLMP-CB15 from Agilent whose light in- 
tensity is peaked at around $470 \mathrm{~nm}$ with a FWHM of $15 \mathrm{~nm}$. These LEDs are used to measure the relative variation of the PMT transit time and dedicated runs of this LED calibration system are customarily taken [2]. This system is used to calibrate the path travelled by the signal starting at the PMT photocathode up to the read-out electronics. The effect of the transmission of the light in water is, however, not addressed by this calibration method.

(3) The Optical Beacons. This system allows the relative time calibration of different OMs to be determined by means of independent and well-controlled pulsed light sources. This system also makes possible to monitor the influence of the water on the light propagation. These Optical Beacons are the subject of this paper and will be described in detail in the following sections.

(4) Several thousands of down-going muon tracks will be detected per day. The hit time residuals of the reconstructed muon tracks can be used to monitor the time offsets of the Optical Modules. This methodology will enable an overall space-time alignment and calibration cross-checks.

Prior to the deployment of the lines, all line elements are verified as functioning correctly in a dedicated dark setup where a time calibration is carried out after the integration of each sector of the line (a sector is one fifth of a line). An optical signal is sent to each OM of every storey. The signal is provided by a Nd-YAG solid state laser that emits intense, short duration light pulses. The light pulse is attenuated before being sent to the OMs. The light is guided through an optical fibre to a 1-to-16 optical splitter. Each of the outgoing fibres is connected to one of the $15 \mathrm{OMs}$ of the sector. The $16^{\text {th }}$ signal is sent to a control module and is used as a time reference. The resulting information from timing calibration in the dark setup is used as the reference for the validation of the in situ timing calibrations. This system is also used to determine the time calibration of the Optical Beacons.

The time calibration depends on the actual location of the OMs which is affected by the slow movements of the lines due to underwater currents. An acoustic positioning system together with a set of compasses and tiltmeters located along the line, provides the OM position with an accuracy of 10$20 \mathrm{~cm}$ which, in addition to the time calibration, is sufficient for the muon track reconstruction [2].

\section{The ANTARES Optical Beacon system}

The Optical Beacon system consists of a series of pulsed light sources distributed throughout the detector. An LED Beacon is composed of several

LEDs, pulsed by dedicated electronic circuits. Those beacons are located, more 
or less, uniformly along every detector line so that their light can illuminate all storeys on the neighbouring lines. The Laser Beacons use a solid state pulsed laser whose light is spread out by a diffuser. Laser Beacons are located at the bottom of a few lines in the so-called Bottom String Socket. The Laser Beacons illuminate mainly the bottom part of the lines and are located in a stationary position. The system of Optical Beacons provides a number of well controlled, pulsed light sources that act as a reference for time calibration of the detector. The system is able to closely monitor all the detector components and the sea water. It allows the monitoring of the absorption and scattering lengths of the sea water.

\section{The LED Beacons}

An LED Beacon contains 36 individual LEDs arranged in groups of six, on six vertical boards (faces) which are placed side by side forming an hexagonal cylinder (figure 1). On each face, one LED points upwards (top $L E D$ ), and the other five LEDs point radially outwards. One of the LEDs that points horizontally is located in the middle of the face (central $L E D$ ) and the remaining four surround it.

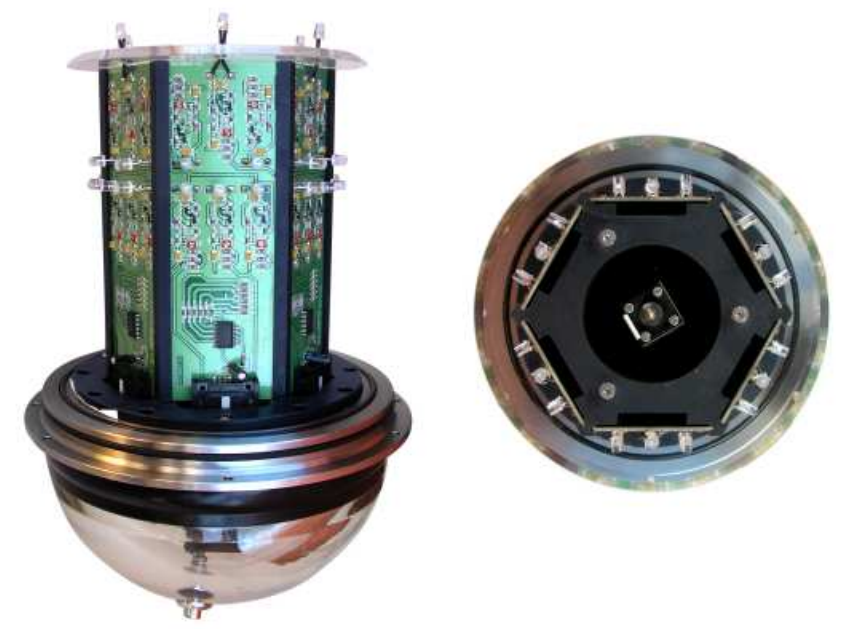

Fig. 1. An LED Beacon as viewed from sideways (left) and from the top (right). Six faces each containing six LEDs are arranged on an hexagonal cylinder. The internal photomultiplier tube is mounted at the centre of the lightguide.

The faces are mechanically fixed to a hollow nylon structure which internally houses a small Hamamatsu H6780-03 photomultiplier tube. This PMT, with 
a photocathode of $8 \mathrm{~mm}$ diameter, has a risetime of $0.8 \mathrm{~ns}$ and a transit time of $5.4 \mathrm{~ns}$ and is used to provide the precise time of emission of the light flash independently of the triggering signal. A flat acrylic disc that acts as a lightguide is fixed to the upper part of the nylon mounting to increase the collection of light. Following Fields and Janowski [9] a conical depression was machined in the centre of the light collecting disc, to direct light into the photomultiplier tube. The edges of the disc were also bevelled at $45^{\circ}$ to improve light collection from the horizontal LEDs.

The lower part of the LED Beacon houses the electronic boards that provide the required operating voltages and enable the actual LED flashing according to externally supplied slow control commands. Each of the six faces can be flashed independently or in combination of different faces. Within a face the top, central and the group of four LEDs can be triggered independently or in combination. This layout allows a distribution close to uniform in the azimuth angle when all the LEDs are flashing. The top LEDs allow the calibration of the OMs in those storeys on the same line above the beacon. The amount of light can be further controlled changing the number of LEDs flashing at a given time while the intensity of the LEDs can also be varied as explained in the following subsection. The possibility of flashing certain faces enables the monitoring of the uncertainty in the time calibration arising from the non-uniformity and anisotropy of the LED Beacons.

\subsection{The pulser system}

The pulser circuit is based on an original design from Kapustinsky et al. [10] that has been modified for ANTARES to include in particular a variable capacitor that enables the synchronisation of the pulses produced by several different circuits (see figure 2). The trigger is provided by a $1.5 \mathrm{~V}$ negative square pulse of a duration of around $150 \mathrm{~ns}$ superimposed on a negative DC bias that can be varied from 0 to $24 \mathrm{~V}$. The DC component charges the capacitor and the rising edge of the differentiated $1.5 \mathrm{~V}$ pulse switches on the pair of transistors, triggering the fast discharge of the $100 \mathrm{pF}$ capacitor through the low impedance path that includes the LED. The parallel inductor develops charge in opposition to the discharging capacitor further reducing its time constant. The level of the DC voltage determines the amount of current through the LED and thus the intensity of the emitted pulse. The layout of the printed circuit board has been designed so as to enable the inclusion of six pulsers on the same face without interference of the distributed triggering signals in the nearby pulser circuits whilst minimizing the difference in the times of arrival of the trigger signal to the different pulsers. Synchronisation of the signals from different LEDs on the same or on different faces is possible by adjusting the variable capacitor in each pulser, this procedure is explained 
in subsection 5.3. When deployed underwater the LED Beacons are operated with a typical trigger frequency of a few Hz. This frequency can be increased to rates up to $1 \mathrm{kHz}$.

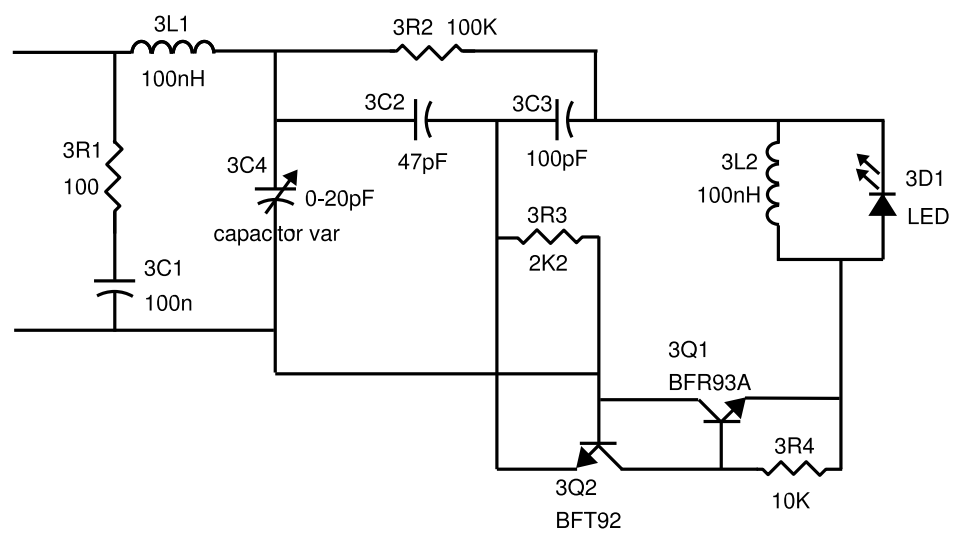

Fig. 2. Diagram of the LED pulser circuit. The variable capacitator (see text) is indicated by label $3 \mathrm{C} 4$.

\subsection{The LEDs}

Different types of LEDs were tested by the ANTARES collaboration in terms of amplitude and risetime of the emitted light pulses. The selected LED was the Agilent HLMP-CB15-RSC00 mode6. This LED has a peak wavelength of $472 \mathrm{~nm}$ with a spectral half-width of $35 \mathrm{~nm}$ according to the specification sheet. The risetime of the LED pulses has been measured and found to be between 1.9 and 2.2 ns. The LEDs were classified in batches according to their risetime and the fastest (1.9-2.0 ns) are used for the top LED locations where the uncertainty in the calibration is dominated by the rise-time and not by the light propagation effects.

To increase the angular occupancy of the light emitted by the LEDs, which was originally restricted to $15^{\circ}$, the caps of the LEDs were machined off. The angular distribution of the emitted light for several depths of cut was measured. The cut lengths tested ranged from 1.5 to $3.5 \mathrm{~mm}$. A cut at $3 \mathrm{~mm}$ was selected, which provides an emission flat within $\pm 10 \%$ for angles up to $35^{\circ}$ and within a factor two up to $55^{\circ}$.

Figure 3 (left) shows the light amplitude as function of the azimuth angle for different cut depths. The right plot in figure 3 shows the time distribution of a single LED pulse.

$\overline{6}$ Agilent Technologies, Inc. Headquarters 395 Page Mill Rd. Palo Alto, CA 94306 United States. 

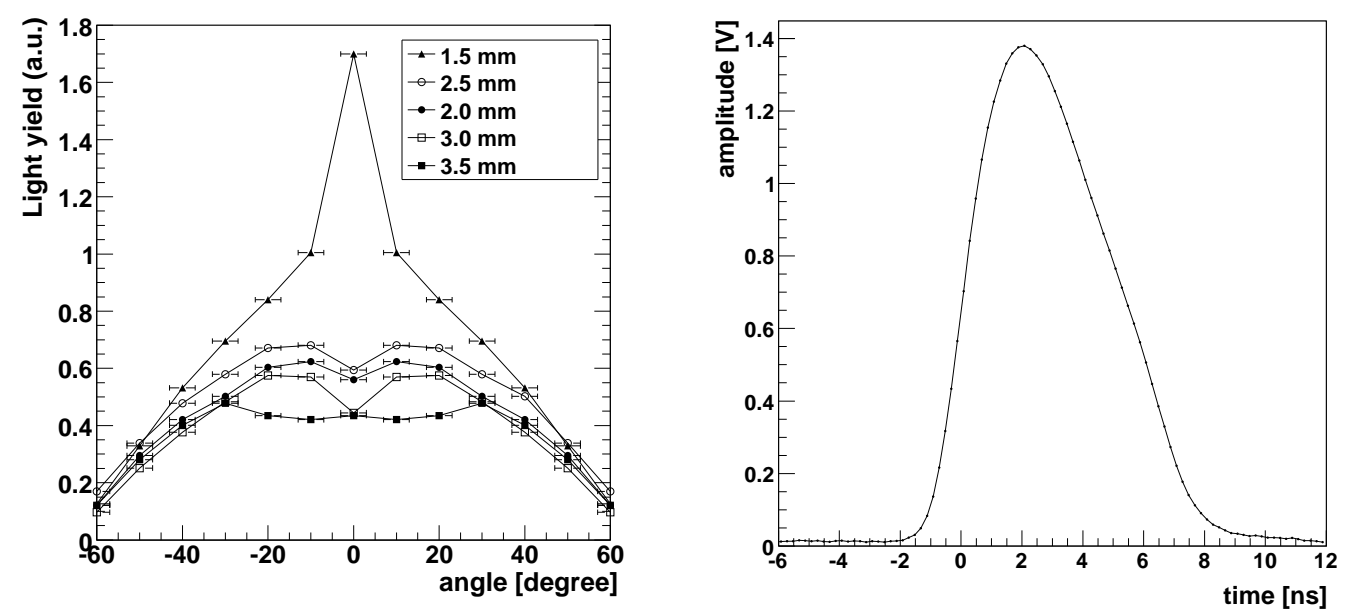

Fig. 3. Left: Light amplitude as a function of the azimuth angle for different cut depths. Right: Timing profile of a single LED pulse.

As mentioned in the previous subsection, the amount of energy per pulse emitted by the LED can be varied by changing the DC voltage from 0 to $24 \mathrm{~V}$. Below $8 \mathrm{~V}$ the amount of light emitted is almost negligible. For a DC level of $24 \mathrm{~V}$ the energy per pulse emitted is at least $150 \mathrm{pJ}$, which corresponds to the emission of approximately $4 \cdot 10^{8}$ photons.

\subsection{Assembly, synchronisation, testing and integration}

Once an LED Beacon is assembled, its 36 LEDs have to be synchronised by tuning the variable capacitor in each of its pulser circuits. This operation takes place in three steps. First the achievable time range of light emission is measured for each LED by adjusting the variable capacitor to its upper and lower limits. Then, taking advantage of the overlap of these time ranges, a common reference time for all LEDs is chosen. Finally, the capacitors are tuned again to reach this reference time. In the left-hand plot of figure 4 the different ranges for each LED pulser for a typical LED Beacon are shown. The full (red) line indicates where the synchronisation common reference time was set and the squares (blue) are the final measured times. The right-hand plot of figure 4 shows the distribution of the final emission times. The typical standard deviation of the emission times is in general a few tens of picoseconds. Following this synchronisation procedure, the beacon undergoes a series of thermal cycles in a climate chamber lasting 48 hours in order to guarantee its stability. The emission times are then remeasured and in exceptional cases some pulsers are re-synchronised.

After synchronisation, the LED Beacon is introduced into the pressure resis- 

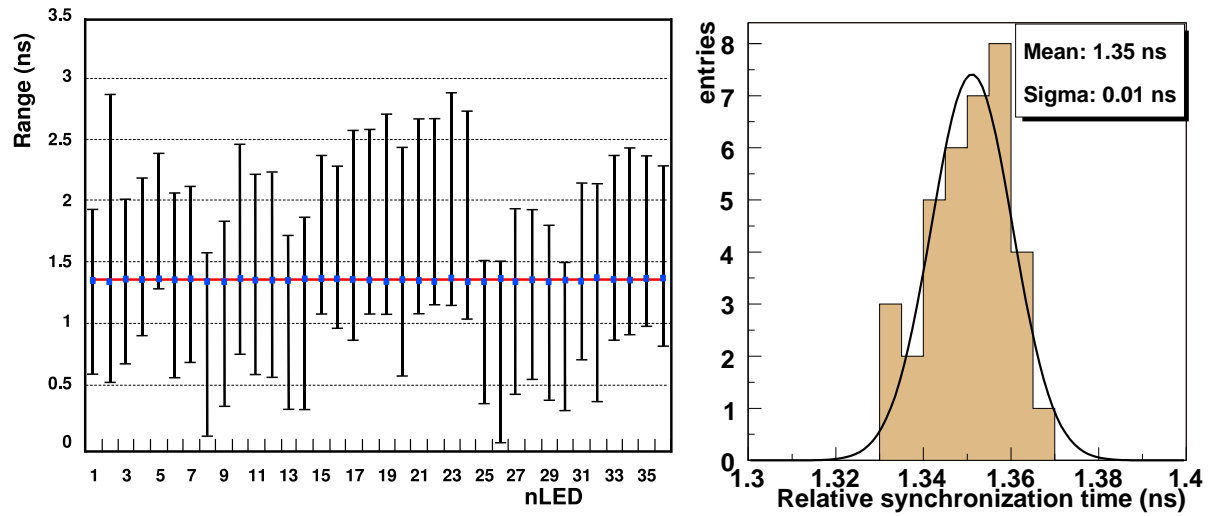

Fig. 4. Left: Emission time ranges of the 36 LEDs of a typical LED Beacon. The horizontal straight line indicates the desired common emission time (reference time), the small squares show the final measured emission times. Right: Distribution of the final emission times.

tant glass vessel that will house it in the sea. This is a cylindrical borosilicate glass container commercially available 7 . It consists of a cylinder and two endcaps (figure 5 left), one of them detachable. All the parts are in transparent glass except for the titanium flanges that hold the two parts together. The overall dimensions of the cylinder plus endcaps are $210 \mathrm{~mm}$ outer diameter and $443 \mathrm{~mm}$ in length. The endcap is supplied with a $22 \mathrm{~mm}$ diameter predrilled hole equipped with a penetrator on the outside of the cylinder and connecting cables on the inside. The LED Beacon is mechanically attached to the detector lines by a collar mounted on the Optical Module Frame (OMF). It is held vertically at a specific location above the triplet of OMs (see figure 5 right) and fixed to the structure combining the 6-fold symmetry of the beacon and the 3-fold symmetry of the OMF so as to minimise shadowing.

As already mentioned, the lines undergo a calibration procedure at the integration sites using a common laser source. The PMT of the LED Beacon is calibrated simultaneously with the PMTs in the OMs using a dedicated fibre from this common source. This calibration facilitates the measurement of their relative shift in the arrival times.

$\overline{7}$ Nautilus Marine Service GmbH, Blumenthalstrasse 15 D-28209 Bremen Germany. 

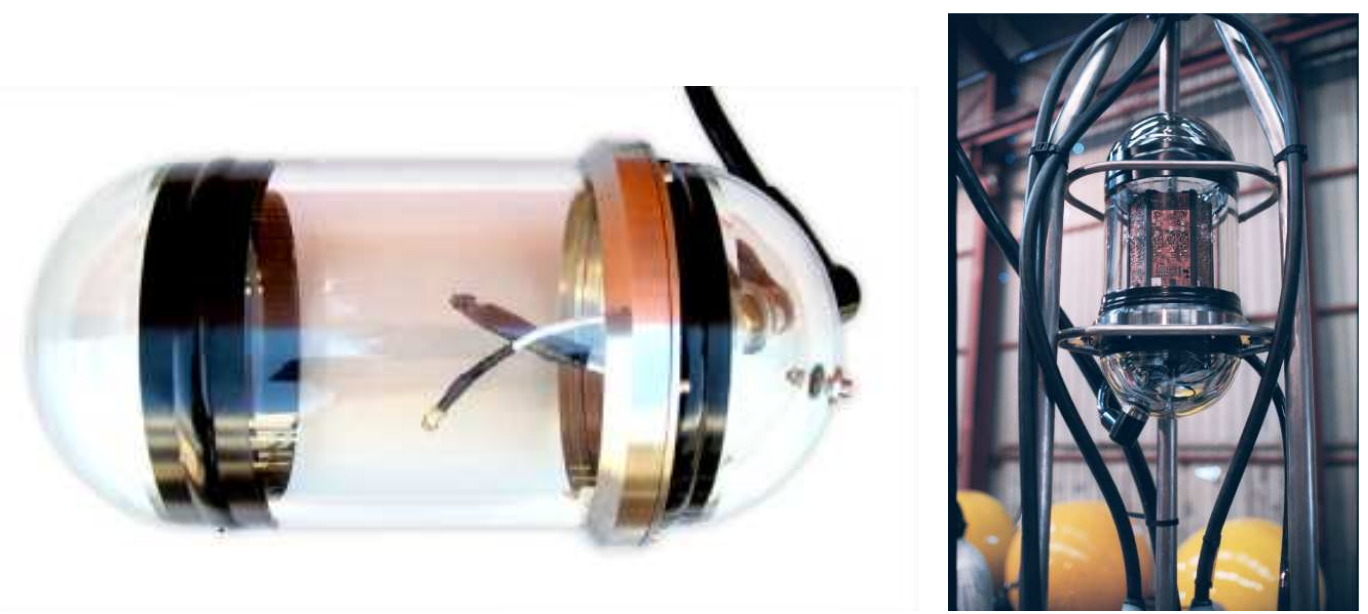

Fig. 5. Left: Example of a borosilicate glass container that houses the LED Beacons. Right: LED Beacon mounted in the Optical Module Frame.

\subsection{Configuration, control and monitoring}

The LED Beacon motherboard controls the operation of the beacon at the most basic level. Its main component is the UNIV1 card, a control board used throughout ANTARES which is based on a 17C756 PIC controller. Communication with the motherboard takes place on an RS485 serial link protocol MODBUS.

The motherboard main functions are 1) to set the DC level supplied to the LEDs (from the $+48 \mathrm{~V}$ input voltage via DC/DC converter) and thus the intensity of the light pulses emitted, 2) to select the faces or group of faces that will be flashed, 3) to select any of the three groups of LEDs or the combination thereof that will flash, 4) to set the PMT gain control voltage, and 5) to monitor the voltage supplied to the LEDs and the ambient temperature as measured by a sensor.

The configuration of all the LED Beacons in the detector is performed through the general RunControl program [11]. Different configurations corresponding to the set of possible faces and groups of LEDs that are requested to flash and the required PMT and LED voltages, are stored in a database. In the configuration stage at the start of a calibration run, a given configuration (a run set-up) can then be selected and downloaded. In addition, a graphical user interface written in Java allows the expert user to communicate directly with the beacons and request their status or change their configuration during the run by generating the necessary Slow Control commands. This latter method is very rarely used during normal data taking, but is employed in the laboratory for test or debugging purposes. 


\section{The Laser Beacons}

The Laser Beacons emit high intensity, short duration pulses of light and will be located at the bottom of a few lines, attached to their Bottom String Socket (BSS), i.e. the mechanical structure that anchors the line to the sea bed. At present, one Laser Beacon has been installed in the so-called MILOM line [2] (see next section). Figure 6] shows a general view of the Laser Beacon and its components. The Laser Beacon points upwards so that the emitted light can reach nearby lines. Since the intensity and time profile of the light pulse are defined by the intrinsic properties of the laser, an extensive study of different laser types was made. The selected model is described in the following subsection.

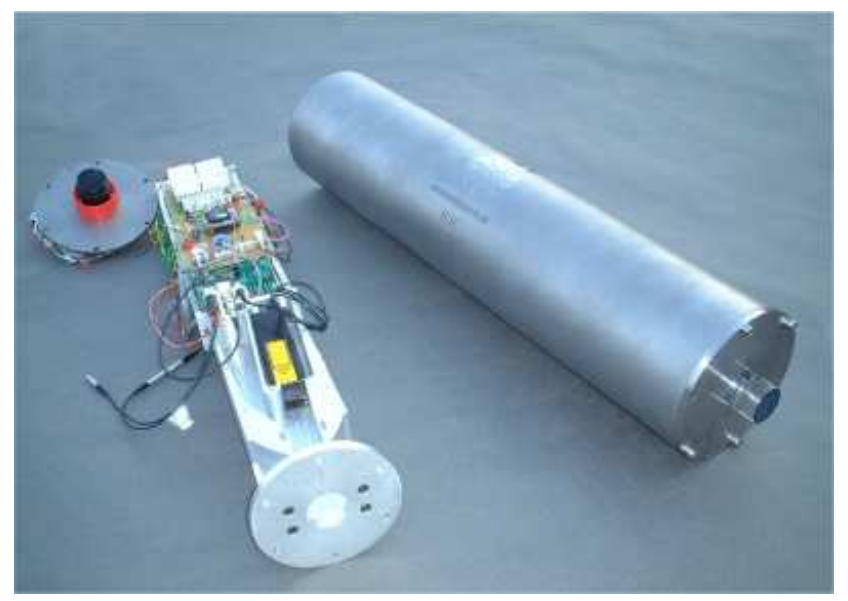

Fig. 6. A Laser Beacon dismantled: On the left the inner mechanics holding the laser head and its associated electronics; on the right the pressure-resistant titanium container that houses the equipment. On the top endplate (right part of the picture) of the container the quartz cylinder that prevents sedimentation effects can be seen.

\subsection{The laser}

The main component of the Laser Beacon is a diode pumped Q-switched NdYAG laser which produces short pulses with a time duration less than $1 \mathrm{~ns}$ (FWHM) and a total energy of $\sim 1 \mu \mathrm{J}$. The laser model selected is the NG10120-120 8 which emits at $532 \mathrm{~nm}$ after frequency doubling of the original Nd-YAG wavelength of $1064 \mathrm{~nm}$.

The laser is very compact. Its head dimensions in $\mathrm{mm}$ are $144 \times 37.4 \times 30$. The laser can be operated in a non-triggered mode at a fixed frequency (around

8 Nanolase, presently part of JDS Uniphase Corp., 430 N. McCarthy Blvd. Milpitas, CA 95035 United States. 
$15 \mathrm{kHz}$ ) or in a triggered mode with a variable trigger frequency. In the latter mode, which is the one being used in ANTARES, the laser is triggered when a TTL signal arrives at the device through a connection in the rear panel of the power supply. Since the laser is passively Q-switched, the delay between the trigger signal and the light pulse emission is of the order of microseconds and the pulse to pulse jitter is of the order of a few hundred nanoseconds. The actual time of laser emission is obtained thanks to a fast photodiode integrated into the laser head.

Once the laser shot is produced, the built-in photodiode sends back a signal which is passed to an ARS chip located in the String Control Module (SCM), the electronics container similar to the LCM located on the BSS. The current that feeds the pumping diode is switched off and the system waits for the next trigger signal.

The power supply delivered with the laser was refurbished in order to comply with the technical requirements of the experiment and, at the same time, to accommodate the whole apparatus into a smaller space. The signal from the photodiode is reshaped electronically to fulfill the constraints imposed by the front-end electronics of the experiment.

In order to characterise the relevant features of the Nd-YAG laser, a thorough study of the main laser parameters was made:

(1) The intrinsic jitter of the Q-switching mechanism gives rise to a jitter in the laser pulse emission time of a few hundred nanoseconds. It was, therefore, necessary to confirm that the time recorded by the internal photodiode was sufficiently accurate for our needs. Several fast external photodiodes (Newport 818-BB-20, Alphalas UPD-200-SP, Hamamatsu S5973-01) were employed to estimate the accuracy in the emission time given by the internal photodiode. Figure 7 (top-left) illustrates the difference in emission time as measured by an external Newport 818-BB-20 photodiode and the internal built-in photodiode. The standard deviation of the distribution is $50 \mathrm{ps}$ (the position of the peak is immaterial, it depends on delays that will be determined by calibration).

(2) The pulse shape was measured using a Hamamatsu streak camera. In figure 7 (top-right) an example of a pulse of the laser as sampled by the streak camera ( 5 ps resolution) is shown. The FWHM of the pulse is determined to be smaller than $0.8 \mathrm{~ns}$. The time shape profile is smooth and not far from Gaussian. As expected, the timing features of the pulse did not change with the three trigger frequencies studied, namely $100 \mathrm{~Hz}$, $1.5 \mathrm{kHz}$ and $10 \mathrm{kHz}$.

(3) Different energy measurements were also performed with a special device 9 capable of measuring the energy of each pulse. As can be seen in

$\overline{9}$ A photodiode head Model PD10 and a laser power meter LaserStar from Ophir, 
figure 7 (bottom-left), after a warm-up period of $\sim 5$ minutes, the energy yield per pulse (close to $1.3 \mu \mathrm{J})$ becomes stable $(< \pm 3 \%)$.
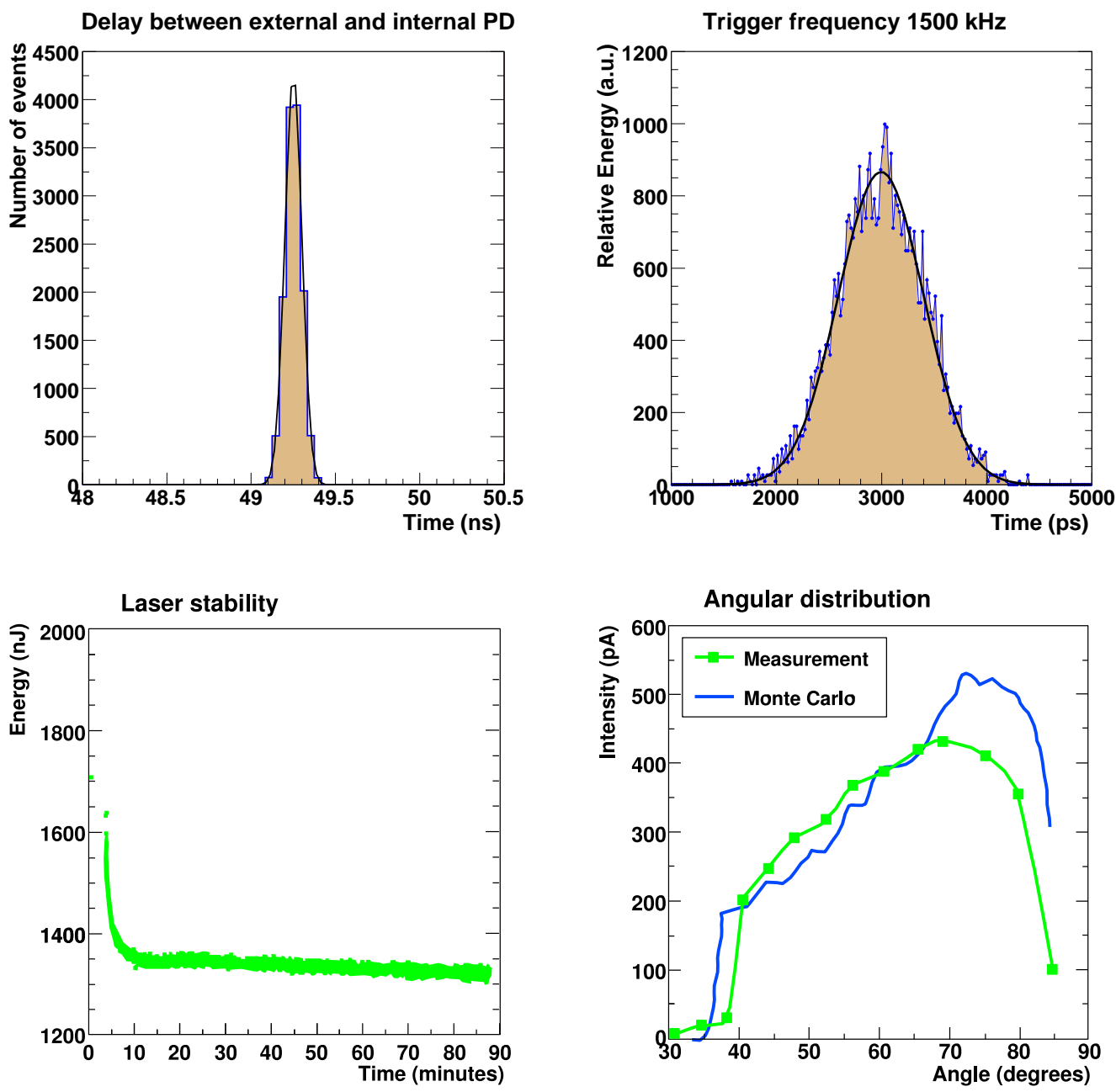

Fig. 7. Top-left: Distribution of the difference of the time of emission of the laser pulse as measured by an external Newport 818-BB-20 photodiode (PD) and the laser built-in photodiode. Top-right: Timing profile of the laser pulse as measured by a Hamamatsu streak camera. Bottom-left: Energy of the laser as a function of time after switch-on. Bottom-right: Angular distribution of the Laser Beacon in water and comparison with Monte Carlo predictions (see section 6.2).

\subsection{The Laser Beacon mounting}

The laser is housed in a cylindrical titanium container $705 \mathrm{~mm}$ in length and $170 \mathrm{~mm}$ in diameter (see figure 6). The bottom endcap holds the penetrator

Laser Measurement Group Ophir Optronics Inc. 260-A Fordham Road Wilmington, MA 01887 United States. 
of the cable connectors. Inside the container, an aluminium inner frame holds the laser and its associated electronics. The laser beam points upwards and leaves the container through an opening in the top end-cap. In this opening there is an optical diffuser comprising a flat disk diffuser 10 with a thickness of $2.2 \mathrm{~mm}$ and a diameter of $25 \mathrm{~mm}$ that spreads light beam out following a cosine distribution, so that the light can reach the surrounding lines.

In order to minimize transmission losses due to underwater sedimentation and biofouling 11 (the laser is more affected by this effect since it is pointing upwards), a quartz cylinder was bonded to the upper surface of the diffuser as can be seen in figure 8 (left). The upper surface of this cylinder is coated with a black, water resistant epoxy layer. The light then leaves the cylinder through the vertical wall where biofouling is negligible (see scheme in figure 8 (right)). Due to Snell's law, the cosine distribution is conserved when the light leaves the cylinder through its vertical wall.

The dimensions of the cylinder were chosen to be $40 \mathrm{~mm}$ in diameter and $47 \mathrm{~mm}$ in length. These dimensions, which together with the refractive index of quartz $(n=1.54)$ and water $(n=1.34)$ determine the maximum and minimum angle of the outgoing light, were selected to maximise the number of storeys illuminated in the closest lines, while taking into account the technical contraints of the cylinder fixing due to the high pressure.

The distribution of the outgoing light was measured by immersing the Laser Beacon into a large water tank that had a PMT on its upper cover and inclining the beacon at different angles with respect to the vertical. In figure 7 (bottom right) the resulting angular distribution is shown together with the result of a simulation. As can be seen, they are in quite good agreement except for high angles, where there are edge effects due to a bevel in the quartz cylinder not reproduced by the Monte Carlo.

The laser diode is fed with $5 \mathrm{~V}$ and $750 \mathrm{~mA}$ supply. As in the case of the LED Beacon, a UNIV1 micro-controller board is used to provide the correct power supply parameters, execute the corresponding switch on and off signals and monitor the measurements given by temperature and humidity sensors inside the container. A thermo-electrical cooling system based on Peltier cells ensures the correct operating temperature of the laser head.

\footnotetext{
$\overline{10}$ ORIEL model 48010, Newport Corporation-Oriel 150 Long Beach lvd. Stratford, CT 06615 United States.

11 the accumulation of micro-organisms, mostly bacteria, on outer surfaces and fallen sediment subsequently adhering to surfaces [8].
} 

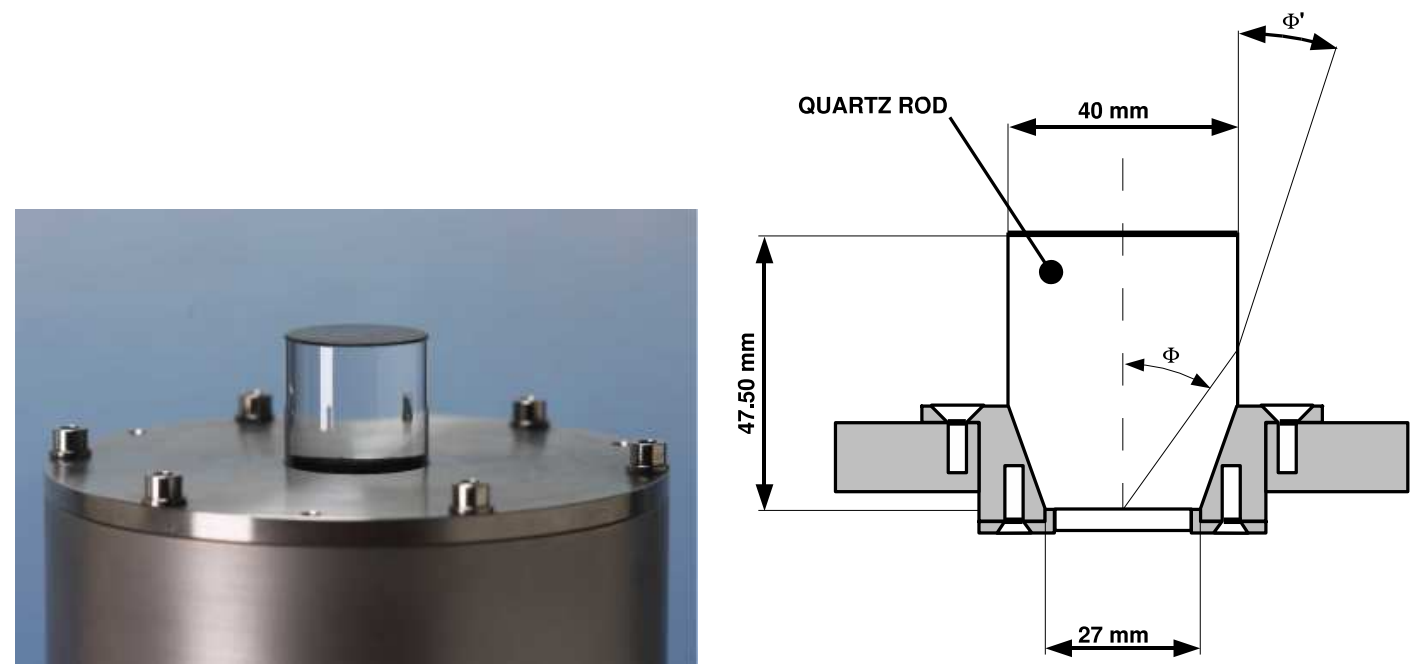

Fig. 8. Left: Upper endplate of the Laser Beacon container with the quartz cylinder used to avoid losses due to biofouling. Right: Schematic path of the outgoing light.

\section{First results of the Optical Beacon system}

An instrumentation line with Optical Modules (MILOM) [2] and the first two complete lines of the detector, Line 1 and Line 2, were already in operation at the ANTARES site at the end of 2006. The three lines are connected to a Junction Box which in turn is linked to the shore by an electro-optical cable. The lines are operated from a control room situated at La Seyne-sur-Mer, near Toulon (France), and data are regularly taken since their connection.

The MILOM has one operating LED Beacon at storey 1 and one Laser Beacon located at the bottom of the line. Lines 1 and 2 are equipped with four LED Beacons at storeys 2, 9, 15 and 21 (numbered from bottom to top). Dedicated data taking runs in which one or several of the Optical Beacons are flashed are regulary performed. Only data from the Optical Beacon system in Line 1 and MILOM are presented here.

Light emitted from the various Optical Beacons is detected by the OMs on the two lines. The distribution of the arrival times of the light at the OMs is presented below. Further details of this analysis will be the subject of a subsequent paper.

Figure 9 shows the distribution of the difference between the time of the signal recorded by each of the three OMs on the third storey of Line 1 and the time of emission of the pulse at the LED Beacon in the storey below as recorded by the beacon's PMT. Together with the time distributions, a Gaussian fit is shown. The values of the Gaussian width are also indicated. The means have been set to zero. 

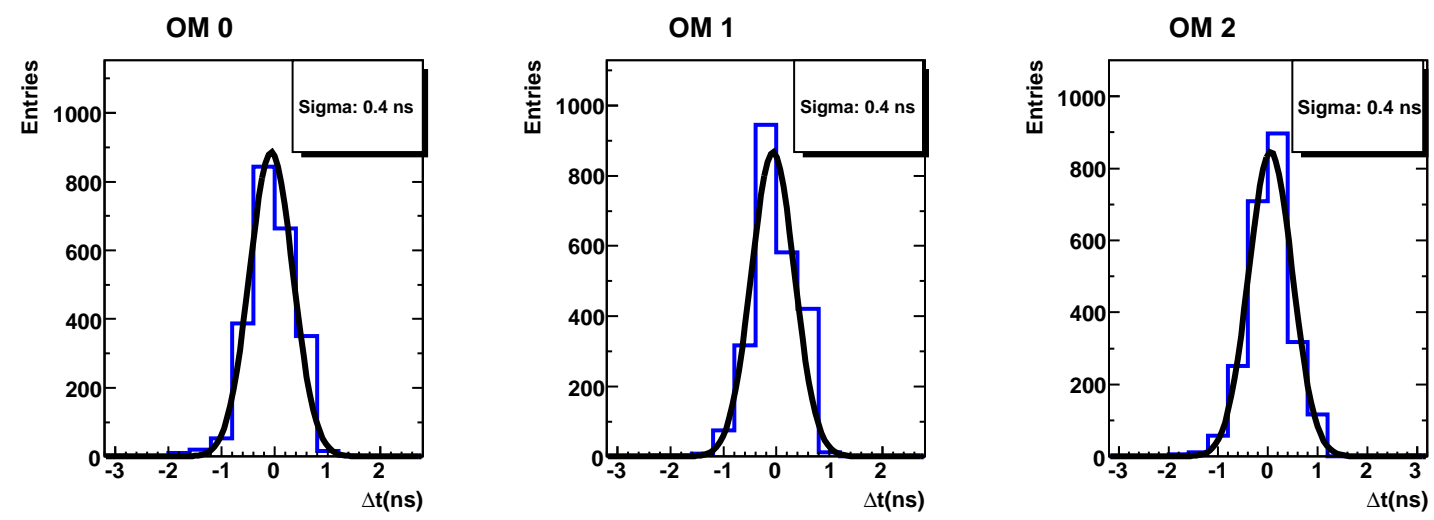

Fig. 9. Distributions of the difference between the time of arrival of the light at the three OMs of storey 3 of Line 1 and the time of emission from the LED Beacon in storey 2 on the same line.

Due to the short distance between the beacon and the OMs $(\sim 13.4 \mathrm{~m})$ and to the high intensity at which the beacon was operated, contributions to the width of the time distributions from the transit time spread in the PMT, from the signal pulse walk, from light scattering in the water and from line movements are all negligible. The width of the time distribution, which is $\sim 0.4 \mathrm{~ns}$, is therefore a measure of the resolution of the read-out electronics which is compatible with the time resolution measured in reference [5].
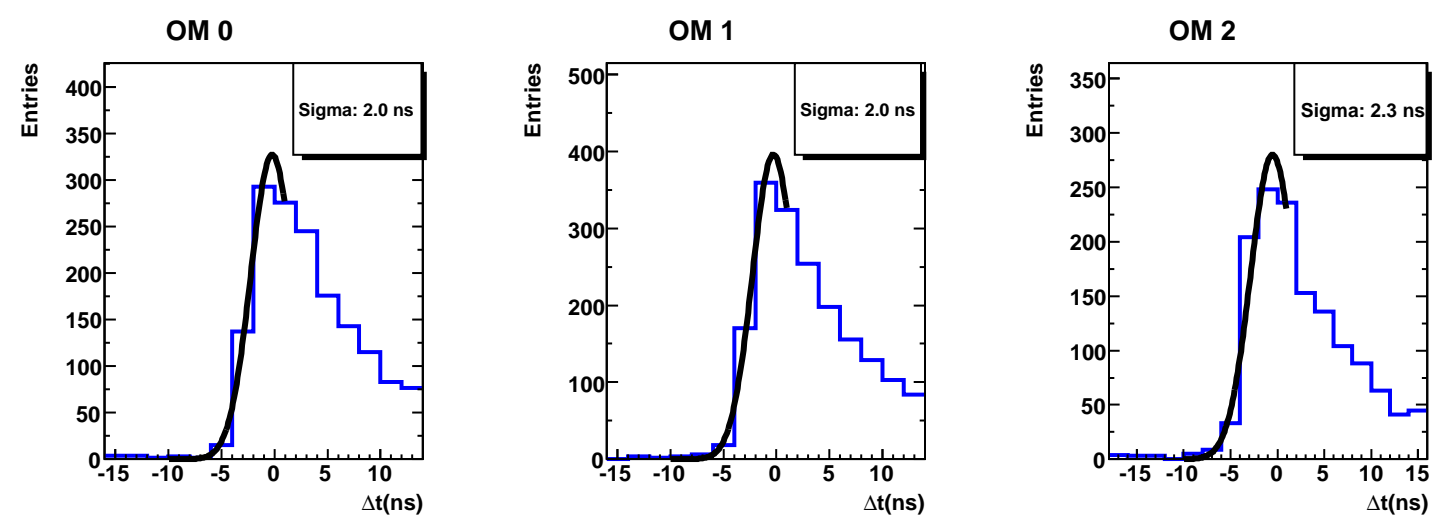

Fig. 10. Distributions of the difference between the arrival time of light at the three OMs of storey 2 of the MILOM and the emission time from the Laser Beacon at the bottom of the same line.

Figure 10 shows the distributions of the difference between the detection time of the signal at the three OMs of the second storey of the MILOM and the emission time of the pulse from the Laser Beacon as provided by the built-in photodiode. In this case, due to the distance between the Laser Beacon and the OMs $(\sim 109 \mathrm{~m})$ a long tail due to scattering is clearly visible. The sigmas of a Gaussian fit to the rising edge of the distributions are shown. The rise-time of the distributions is the outcome of the convolution of the rise-time of the laser pulse itself and the effect of scattering on the early photons. No correction has 
been applied to the data. The position of the peaks of the distributions have been set to zero.

The Optical Beacon system is also used for time calibration between different lines. Figure 11 shows the distribution of the difference between the detection time of the signal at the OMs in Line 1 third floor and the emission time of the light by the LED Beacon located in the MILOM first storey. The distance between the triplet and the Optical Beacon is about $80 \mathrm{~m}$. Due to the orientation of the triplet in the storey, one of the OMs looks in the opposite direction to the MILOM and therefore can only detect scattered photons which explains the tail in the time distribution. The results of the Gaussian fits to the full distributions, or its rising edge when scattering is present, are shown.
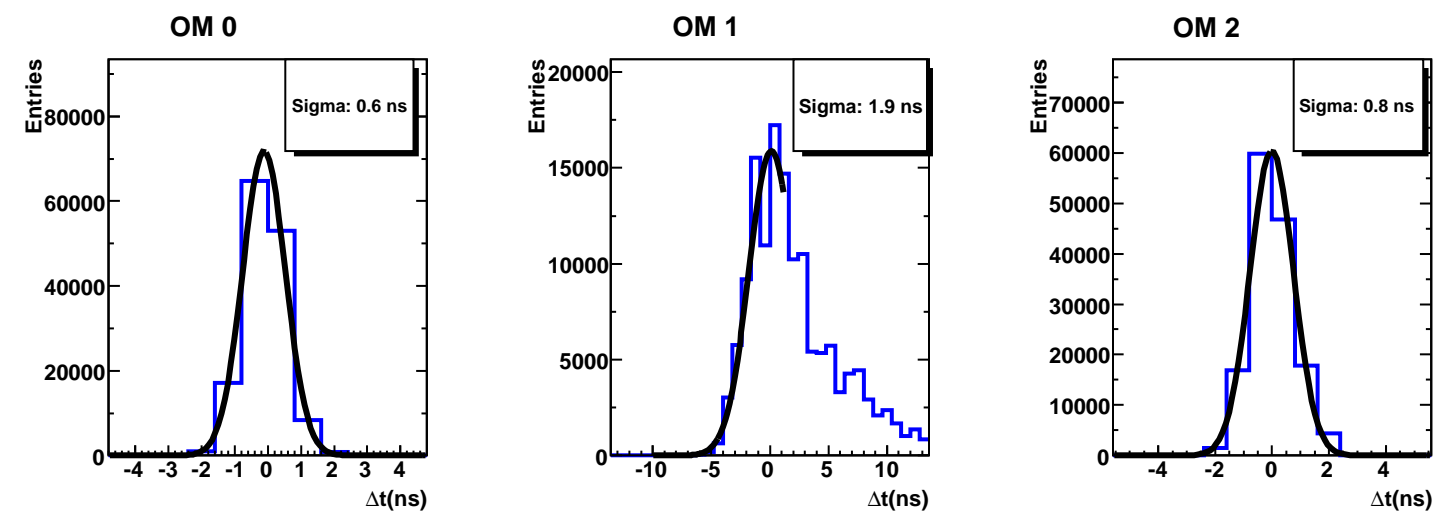

Fig. 11. Distributions of the difference between the arrival time of the light at the three OMs in Line 1 storey 3 and the emission time from the LED Beacon in the first storey of the MILOM.

\section{Summary and conclusions}

In order to ensure the high angular resolution that is aimed by ANTARES, an exhaustive R\&D study to develop different time calibration systems has been carried out. These systems must guarantee a relative time resolution at the sub-nanosecond level between the Optical Modules of the detector. In this article the main features of the Optical Beacon calibration system of the ANTARES detector have been described. This system uses short light pulses produced by LEDs or lasers to measure time differences and therefore calibrate the timing of the detector. The design and construction of these Optical Beacons together with the tests performed in the laboratory have been described. The first results of some of these devices located at $2500 \mathrm{~m}$ depth have been given. With these calibration systems it has been demonstrated that, in situ, ANTARES achieves a sub-nanosecond time resolution. 


\section{Acknowledgements}

The authors acknowledge the financial support of the funding agencies: Centre National de la Recherche Scientifique (CNRS), Commissariat à l'Energie Atomique (CEA), Commission Européenne (FEDER fund and Marie Curie Program), Région Alsace (contrat CPER), Région Provence-Alpes-Côte d'Azur, Département du Var and Ville de La Seyne-sur-Mer, in France; Bundesministerium für Bildung und Forschung (BMBF), in Germany; Istituto Nazionale di Fisica Nucleare (INFN), in Italy; Stichting voor Fundamental Onderzoek der Materie (FOM), Nederlandse organisatie voor Wetenschappelijk Onderzoek (NWO), in The Netherlands; Russian Foundation for Basic Research (RFBR), in Russia; Particle Physics and Astronomy Research Council (PPARC), in U.K.; National Authority for Scientific Research (ANCS) in Romania; Ministerio de Educación y Ciencia (MEC), in Spain.

\section{References}

[1] E. Aslanides et al., the ANTARES proposal, (1999), astro-ph/9907432, http://antares.in2p3.fr.

[2] J. A. Aguilar et al., ANTARES Collaboration, Astroparticle Physics 26 (2006) 314.

[3] P. Amram et al., ANTARES Collaboration, Nucl. Instr. and Meth. Phys. Res. A484 (2002) 369.

[4] J. A. Aguilar et al., ANTARES Collaboration, Nucl. Instr. and Meth. Phys. Res. A555 (2005) 132.

[5] F. Feinstein, ANTARES Collaboration, Nucl. Instr. and Meth. Phys. Res. A504 (2003) 258.

[6] J. A. Aguilar et al., ANTARES Collaboration, Astroparticle Physics 23 (2005) 131.

[7] A. Romeyer, Étude de la sensibilité du détecteur ANTARES à un flux diffus de neutrinos cosmiques de haute énergie, Thesis in Université Denis Diderot - Paris VII. http://antares.in2p3.fr/Publications/thesis/2003/Alain-Romeyer-phd.pdf.

[8] P. Amram et al., ANTARES Collaboration, Astroparticle Physics 19 (2003) 253.

[9] T. Fields and D. Jankowski, Nucl. Instr. and Meth. Phys. Res. 215 (1983) 131.

[10] J. S. Kapustinsky et al., Nucl. Instr. and Meth. Phys. Res. A241 (1985) 612.

[11] J. A. Aguilar et al., ANTARES Collaboration, Nucl. Instr. and Meth. Phys. Res. A570 (2007) 107. 